\title{
Innervation of the Rat Anterior Abdominal Wall as Shown by Modified Sihler's Stain
}

\author{
Engin Calguner ${ }^{a}$ Deniz Erdogan $^{b}$ Cigdem Elmas $^{b}$ Meltem Bahcelioglu ${ }^{a}$ \\ Rabet Gozila ${ }^{a}$ Mehmet Suhan Ayhan ${ }^{c}$ \\ Departments of a Anatomy, ${ }^{b}$ Histology and Embryology, and ${ }^{c}$ Plastic and Reconstructive Surgery, \\ School of Medicine, University of Gazi, Ankara, Turkey
}

\section{Key Words}

Sihler's stain technique $\cdot$ Abdominal wall muscle innervation

\begin{abstract}
Objective: The purpose of this study was to use the modified Sihler's staining technique to demonstrate detailed distribution of the rat anterior abdominal wall nerves and test the value of Sihler's technique in demonstrating such a complex muscle-nerve relationship. Materials and Methods: The anterior abdominal walls of 5 Wistar rats were isolated by making a deep incision from the costal arches on each side down to the inguinal region and processed using a modified Sihler's stain technique. Results: This technique was successfully applied to visualize the innervation of the anterior abdominal wall muscles of the rat. The segmental nerves of $T_{6}-L_{1}$ and their terminal branches were shown and possible motor and sensory fibers identified. Conclusions: This technique is valuable in understanding the complex nature of final branching of the nerve endings, and it may be useful for studying experimental nerve models.
\end{abstract}

\section{KARGER}

Fax +4161306 1234 E-Mail karger@karger.ch www.karger.com

\section{Introduction}

Anatomical dissection has been the most common method for tracing nerve fibers to their fine intramuscular terminal branches. The intramuscular branches, however, cannot easily be revealed by direct dissection. Such dissections, even under the microscope can damage muscle fibers and disrupt the normal anatomical relationship between muscle and nerve fibers. Computer reconstruction of serial histological sections has also been used as an alternative method for tracing nerve supply. Also, gold chloride staining has been applied by many to visualize nerves. Even with this method, nerve fibers were visible only superficially, and tracing their branches in deep sections was not possible $[1,2]$.

Charles Sihler [3] introduced a method for staining nerve spindles in 1895 , but the work remained largely forgotten until recently. The technique makes whole specimens transparent so that the intramuscular nerve supply appears in its original three-dimensional state. Consequently, tracing the complex structure of terminal nerve branches becomes easier. Many researchers have reported modifications of Sihler's technique for identifying innervations to various organs [4-7].

The muscles of the rat anterior abdominal wall, namely the external oblique, internal oblique and transverse, form three thin aponeurotic layers which eventually en- 
velope the rectus abdominis ventrally, similarly to those of humans. These muscles are supplied by lower thoracic spinal nerves, iliohypogastric and ilioinguinal nerves. There are 34 spinal nerves in the rat: 8 cervical, 13 thoracic, 6 lumbar, 4 sacral and 3 caudal. Morphologically, the pattern of anterior abdominal wall innervations is like that of humans. However, a communicating branch of the 13th thoracic nerve frequently joins with the first lumbar nerve. It divides into two branches; a superior one (the iliohypogastric nerve), which may be joined by a branch from the 13th thoracic, and an inferior one (the ilioinguinal nerve), which is somewhat variable. In some cases, these two branches may form a single trunk for a short distance, or the inferior branch may unite with a branch from the second lumbar nerve $[8,9]$. The iliohypogastric nerve first gives cutaneal branches (lateral and ventral) for the anterior abdominal wall, then follows its course and eventually divides into cranial and caudal branches [10]. The muscles of the anterior abdominal wall and their nerve supplies are fairly complex and the gross dissections of branches of related muscles are somewhat difficult [11]. Furthermore, when tracing nerve branches deeper into the muscles they innervate, preserving one branch often necessitates unwanted destruction of other nerves to the same structure [12]. We designed this study in order to demonstrate detailed distribution of the anterior abdominal wall nerves of the rat and tested the value of Sihler's technique in such a complex muscle-nerve relationship.

\section{Materials and Methods}

Five Wistar rats were killed by intravenous injection of $1-2 \mathrm{mg}$ $\mathrm{kg}^{-1}$ sodium pentobarbital. The anterior abdominal walls of the rats were carefully isolated by making a deep incision from the costal arches on each side down to the inguinal region and processed using a modified Sihler's technique. The chemical reagents used in this study were obtained from Sigma and Merck Chemical Co. Approval for this experimental study protocol was obtained from the Local Ethical Committee for Animal Studies.

\section{Tissue Preparation}

The specimens were fixed by immersion in $10 \%$ unbuffered formaldehyde for 1 month. The fixed specimens were washed under running water for $1 \mathrm{~h}$ and then macerated in 3\% $\mathrm{KOH}$ solution (3 drops of $3 \% \mathrm{H}_{2} \mathrm{O}_{2}$ added to $100 \mathrm{ml}$ of the $\mathrm{KOH}$ ) for at least 3 weeks. The $\mathrm{KOH}$ solution was changed daily. The process was considered complete when the specimens appeared transparent or translucent.

The macerated specimens were decalcified using Sihler's solution I (1:1:6 glacial acetic acid:glycerin: $1 \%$ aqueous chloral hydrate) for 2 weeks, with weekly change of the solution, until the specimens became soft.

Rat Anterior Abdominal Wall Innervation Shown by Sihler's Stain

\section{Staining}

The decalcified specimens were transferred into Sihler's solution II (1:1:6 Ehrlich's hematoxylin:glycerin:1\% aqueous chloral hydrate) for 5 weeks. Excess stain was removed from the specimens by placing them in Sihler's solution I. The solution was changed each time it turned purple. This procedure was repeated for 2 days until the muscles were stained light purple and the nerves, a dark color [13].

After excess stain has been removed, the specimens were washed under running water for an hour and then immersed in $0.05 \%$ lithium carbonate solution for approximately $1 \mathrm{~h}$. The specimens were cleared in increasing concentrations of glycerin $(40,60,80$ and $100 \%)$ containing a few thymol crystals. The specimens were left in each of the solutions for a day until excess stain was removed.

\section{Photography}

Glycerin-preserved specimens were trimmed carefully using a binocular microscope to display the nerves, and photographs were taken using Nikon Coolpix 995.

\section{Results}

Of the 13 intercostal nerves, the 6-13th individual nerves were followed segmentally to reach the rectus sheath. They were easily traced from lateral (dark-color branches) to medial (light-color branches), as seen in figure 1. As they reached near midline, each individual nerve first gave off lateral and then ventral abdominal branches. The light-colored fine short branches were visible reaching to the abdominal muscles. It was clearly noticeable that the ventral abdominal cutaneal branches traveled to midline by piercing the rectus sheath (fig. 1).

The branches of $T_{6}$ and $T_{7}$ nerves innervated the skin superior to the umbilicus, $\mathrm{T}_{8}$ around the umbilicus and $\mathrm{T}_{9-13}$ and $\mathrm{L}_{1}$ inferior to the umbilicus. The light-colored fine short branches within the rectus sheath were motor as well as sensory nerves to the rectus abdominalis muscle. The nerve traveling laterally upwards along the linea alba was thought to be cranial iliohypogastric nerve (fig. 1).

\section{Discussion}

In recent years, several investigators have successfully applied Sihler's staining technique to demonstrate detailed innervations to various muscles in rabbits [13-16], dogs [17, 18] and humans [19-21].

In this study, in order to highlight the uniqueness of Sihler's staining technique for demonstrating intramuscular nerve fibers, we selected rat anterior abdominal wall to identify and name the nerve branches between the lay- 
Fig. 1a, b. Abdominal muscles seen from above. $\mathrm{U}=$ Umbilicus; $\mathrm{RS}$ = rectus sheath; ICN $\left(\mathrm{T}_{6}-\mathrm{T}_{13}=\right.$ intercostal nerves (ventral ramie); $\mathrm{LACB}=$ lateral abdominal cutaneal branch; $\mathrm{VACB}=$ ventral abdominal cutaneal branch; $\mathrm{MB}$ = muscular branches; $\mathrm{CIN}=$ cranial iliohypogastric nerve.
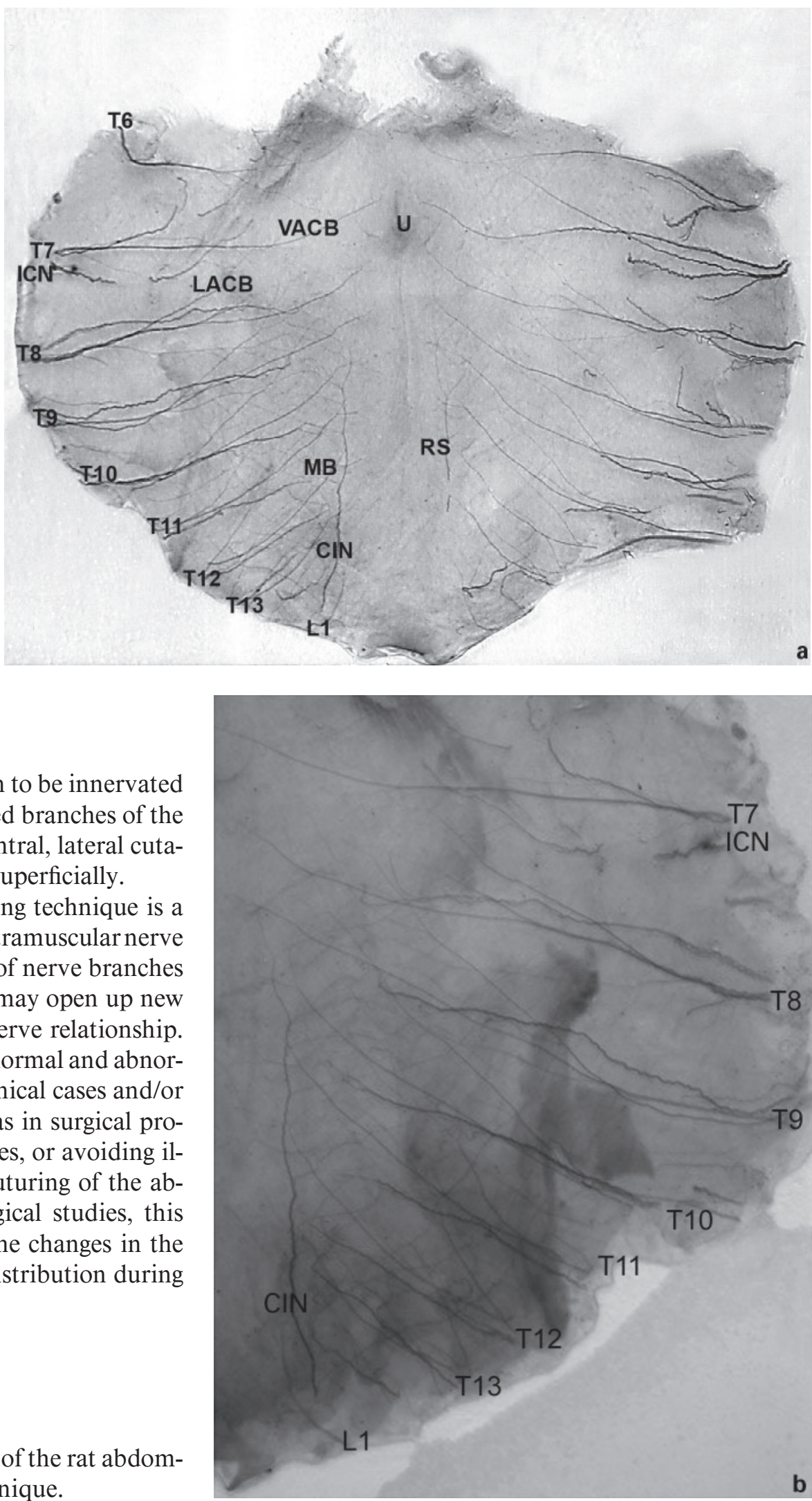

ers of muscles. The muscles were shown to be innervated by $\mathrm{T}_{6-13}$ and $\mathrm{L}_{1}$, segmentally. The mixed branches of the concerned nerves were identified as ventral, lateral cutaneal ramie, and they were distributed superficially.

As seen in this study, Sihler's staining technique is a suitable technique for demonstrating intramuscular nerve fibers and it enables the identification of nerve branches and terminal endings. This technique may open up new horizons for the study of the muscle-nerve relationship. It would be valuable in understanding normal and abnormal functions of any given organ in clinical cases and/or experimental research models as well as in surgical procedures, i.e. suturing abdominal muscles, or avoiding iliohypogastric nerve trapping during suturing of the abdominal layers [22-25]. In embryological studies, this technique could also help determine the changes in the morphological structure of the nerve distribution during embryonic development.

\section{Conclusion}

We presented the nerve distribution of the rat abdominal wall in detail by Sihler's stain technique. 


\section{References}

1 Serlin DM, Schieber MH: Morphologic regions of the multitendoned extrinsic finger muscles in the monkey forearm. Acta Anat 1993;146: 255-266.

-2 Richmond FJR, MacGillis DRR, Scott DA: Muscle-fiber compartmentalization in cat splenius muscles. J Neurophysiol 1985;53:868885.

3 Sihler C: Ueber Muskelspindeln und intramuskuläre Nervenendigungen bei Schlangen und Fröschen. Arch Mikros Anat Entwickl 1895;46:709-723.

$\checkmark 4$ Wharton LR: A technique for studying the innervation of organs. Anat Rec 1937;67:469475.

$\checkmark 5$ Williams TW: A technique for the gross differential staining of peripheral nerves in cleared vertebrate tissue. Anat Rec 1943;86:189-194.

$\checkmark 6$ Freihofer WC: The Sihler technique of staining nerves for systematic study especially of fishes. Copeia 1966;52:470-475.

7 Wu BL, Sanders I, Mu L, Biller HF: The human communicating nerve. Arch Otolaryngol Head Neck Surg 1994;120:1321-1328.

8 American Philosophical Society: Anatomy of the Rat. New York, Hafner, 1963.

9 Chiasson RB: Laboratory Anatomy of the White Rat. New York, McGraw-Hill, 1994.

10 Department of Veterinary Anatomy: Nomina Anatomica Veterinaria. New York, Cornell University Press, 1994.

-11 Monkhouse WS, Khalique A: Variations in the composition of the human rectus sheath: a study of the anterior abdominal wall. J Anat 1986;145:61-66.
12 Ryan S, NcNicholas WT, O'Regan RG, Nolan $P$ : Intralaryngeal neuroanatomy of the recurrent laryngeal nerve of the rabbit. J Anat 2003; 202:421-430.

13 Gözil R, Kadioglu D, Calguner E, Erdogan D, Bahcelioglu M, Elmas C: Branching patterns of rabbit oculomotor and trochlear nerves demonstrated by Sihler's stain technique. Biotech Histochem 2002;77:21-25.

14 Liu J, Kumar VP, Shen Y, Lau HK, Pereira BP, Pho RWH: Modified Sihler's technique for studying the distribution of intramuscular nerve branches in mammalian skeletal muscle. Anat Rec 1997;247:137-144.

15 Kierner AC, Zelenka I, Burian M: How do the cervical plexus and the spinal accessory nerve contribute to the innervation of the trapezius muscle? Arch Otolaryngol Head Neck Surg 2001; 127:1230-1232.

16 Peker T, Turgut HB, Gülekon N, Anıl A: Demonstration of the nerve distribution of the masticatory muscles in rabbits (Oryctolagus cuniculus). Anat Histol Embryol 2001;30:225-229.

$\checkmark 17$ Mu L, Sanders I: Neuromuscular specializations of the pharyngeal dilator muscles. II. Compartmentalization of the canine genioglossus muscle. Anat Rec 2000;260:308-325.
18 Drake III W, Li Y, Rothschild MA, Wu BL, Biller HF, Sanders I: A technique for displaying the entire nerve branching pattern of a whole muscle: results in 10 canine posterior cricoarytenoid muscles. Laryngoscope 1993; 103:141-148.

$19 \mathrm{Mu}$ L, Sanders I, Wu BL, Biller HF: The intramuscular innervation of the human interarytenoid muscle. Laryngoscope 1994;104:33-39.

20 Sanders I, Mu L: Anatomy of the human internal superior laryngeal nerve. Anat Rec 1998; 252:646-656.

$21 \mathrm{Mu}$ L, Sanders I: Sensory nerve supply of the human oro- and laryngopharynx: a preliminary study. Anat Rec 2000;258:406-420.

22 Burger JW, Van't Riet M, Jeekel J: Abdominal incisions: techniques and postoperative complications. Scand J Surg 2002;91:315-321.

23 Duchateau J, Declety A, Lejour M: Innervation of the rectus abdominis muscle: implications for rectus flaps. Plast Reconstr Surg 1988; 82:223-228.

24 Mandelkow H, Loeweneck H: The iliohypogastric and ilioinguinal nerves. Distribution in the abdominal wall, danger areas in surgical incisions in the inguinal and pubic regions and reflected visceral pain in the dermatomes. Surg Radiol Anat 1988;10:145-149.

25 Yan L, Zhong S: Applied anatomy of the transverse rectus abdominis musculocutaneous (TRAM) flap in relation to the eleventh or twelfth thoracic nerve. Zhonghua Zheng Xing Wai Ke Za Zhi 2000;16:81-83. 